\title{
Long-term effects on the liver
}

\author{
PETER J. SCHEUER \\ From the Department of Histopathology, Royal Free Hospital, London
}

Two or three decades ago chronic liver injury due to drugs was scarcely recognized. Today a wide range of diseases, previously thought to be due to 'natural' causes, is attributed to therapy, although final proof of the cause is often difficult or impossible to achieve. In addition, new drugs have been produced which have subsequently been shown to have hepatotoxic effects. These may only be manifest in a very small proportion of patients, and can even, as in the case of methotrexate, be avoided by judicious dose regimens; demonstration of a hepatotoxic effect therefore does not necessarily mean that an important and irreplaceable drug must be withdrawn from use. This paper deals only with therapeutic and prophylactic drugs. Other toxic substances, notably ethanol and plant poisons such as aflatoxin, almost certainly play a much larger role in chronic liver disease in man but will not be discussed here.

\section{Classification of Drug-induced Liver Injury}

A distinction is drawn between predictable and unpredictable injury, and while this may not reflect fundamental differences in the mode of action of drugs on the liver, the classification is useful. Drugs may act predictably in the sense that all patients receiving a sufficiently large dose over a sufficiently long period will develop a lesion. An example of this is the liver-cell necrosis produced by paracetamol (Davidson and Eastham, 1966). Predictable injury can often be reproduced in laboratory animals if the right animal and dose schedule are discovered. Other injuries are unpredictable, in that only a small proportion of patients on a particular drug will develop a lesion, and it is not possible to identify this minority beforehand. An example of unpredictable injury is the acute cholestatic syndrome produced by chlorpromazine (Ishak and Irey, 1972). Unfortunately such lesions, in contrast to the predictable ones, cannot so far be produced in animals; this has hampered the study of drug reactions and the definite incrimination of individual drugs. It will be seen from the discussion below that examples of chronic drug injury can be found in both the predictable and unpredictable group.
Chronic Effects of Single Episodes of Acute Liver Injury

Single episodes of acute necrosis, such as follow paracetamol, carbon tetrachloride or mushroom poisoning, are only rarely followed by cirrhosis. If the liver damage is sufficiently severe and the architectural integrity of the lobules threatened, collapse will occur but is more likely to lead to irregular scarring then to true cirrhosis with its characteristic tendency to progress and evolve. This is in keeping with the observation that patients with non-fatal massive hepatic necrosis complicating acute viral hepatitis tend to make a complete clinical recovery. Very rarely acute jaundice due to chlorpromazine becomes chronic in the absence of continued therapy (Read, Harrison, and Sherlock, 1961; Walker and Combes, 1966) but complete clinical and histological recovery is far more usual.

It thus seems that repeated or prolonged exposure to a drug is normally necessary for the production and maintenance of a chronic liver lesion. This is clearly more likely to occur when the liver disease is clinically silent or when the role of a drug is not suspected.

\section{Range and Pathogenesis of Chronic Liver Lesions due to Drugs}

An extremely wide variety of long-term effects has been attributed to drugs, ranging from repeated episodes of cholestasis to cirrhosis and neoplasia. In some cases the chronic disease is a sequel to an identified acute liver injury (Schweitzer and Peters, 1974); more often the liver disease is already well established by the time of diagnosis, in which case the relationship between therapy and disease is difficult to establish. Histological features, response to withdrawal of the drug, and exacerbation on re-exposure may all help to incriminate a drug.

The following categories of chronic or repeated drug injury can be recognized:

HEPATOCELLULAR DISEASE

A small number of drugs has been shown to cause 
a clinical and histological picture of active chronic hepatitis closely resembling the 'natural' disease (fig 1). These include the laxative oxyphenisatin (Reynolds, Peters, and Yamada, 1971) and methyldopa (Goldstein, Lam, and Mistilis, 1973). Other agents held responsible for isolated instances of chronic hepatitis or cirrhosis include inorganic arsenates (Franklin, Bean, and Hardin, 1950), isoniazid (Merritt and Fetter, 1959), halothane (Klatskin and Kimberg, 1969), phenobarbitone (Klatskin, 1969), salicylates (Seaman, Ishak, and Plotz, 1974) and sulphonamides (Tönder, Nordöy, and Elgjo, 1974).

It should be stressed that with most of the above drugs chronic liver disease is a rare consequence and the effect is unpredictable. In the case of oxyphenisatin, the unpredictable nature of the effect has been disputed (Goldstein et al, 1973), and the action of salicylates and arsenic could be dose-dependent. Most of the drugs cited can also give rise to an acute hepatitis similar to viral hepatitis.

The story of oxyphenisatin jaundice illustrates the difficulty of identifying aetiological agents in chronic liver disease. McHardy and Balart (1970) and Reynolds, Lapin, Peters, and Yamahiro (1970)

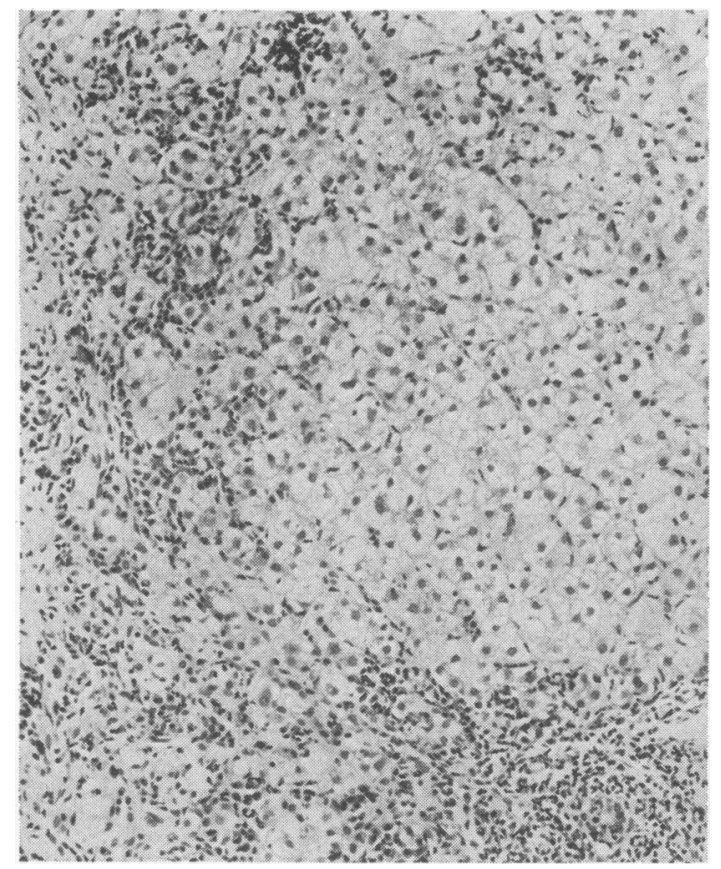

Fig 1 Active chronic hepatitis attributed to oxyphenisatin. There is heavy inflammatory infiltration and piecemeal necrosis. Needle biopsy of liver. Haematoxylin and eosin. $\times 135$. reported on patients with acute hepatitis for which a viral aetiology was assumed until it was observed that further episodes of jaundice and abnormal liver function tests were associated with the taking of a laxative containing oxyphenisatin. Some patients had taken this for over a year before the onset of symptoms, and the drug was not at first suspected. Subsequently it was noted that some patients improved rapidly when the drug was withdrawn. Liver biopsies showed a picture somewhat like that of viral hepatitis, although Reynolds et al (1970) noted that the swelling of liver cells and disorganization of liver-cell plates characteristic of this disease were lacking. Oxyphenisatin may have been responsible for earlier outbreaks of chronic active hepatitis (see fig 1), and Fog (1949) had noted a strong association between laxative intake and chronic hepatitis as long ago as two decades before oxyphenisatin was finally incriminated.

The antimetabolite methotrexate is capable of causing cirrhosis without, as a rule, giving rise to the histological and clinical picture of active chronic hepatitis, or to an acute hepatitis of viral type. This drug is now given over long periods of time to patients with severe psoriasis as well as to patients



Fig 2 Cirrhosis following long-term methotrexate therapy. A small nodule is outlined by a slender septum. There is mild fatty change. Needle biopsy of liver. Haematoxylin and eosin. $\times 135$. 
with malignant disease. A common finding on liver biopsy is a non-specific picture with fatty change, mild portal and parenchymal inflammation and increased variation in liver-cell appearances. This picture can, however, also be found in psoriatic patients who have not received methotrexate. With increasing length of treatment in the form of frequent small oral doses, fibrosis develops in the form of septa extending from the portal tracts. Cirrhosis finally ensues (fig 2), and it has been shown that the duration of treatment in patients with serious liver disease was significantly longer than in those with the milder, non-specific picture (Dahl, Gregory, and Scheuer, 1971). The majority of patients treated for two years or more had severe fibrosis or cirrhosis. Methotrexate injury is therefore an example of a predictable, dose-dependent lesion. Fortunately the hepatotoxic effects can be greatly reduced by the use of intermittent therapy (Dahl, Gregory, and Scheuer, 1972; Cooperative Study, 1973). Liver biopsy is important in the assessment of patients on long-term methotrexate, since liver function tests may give a falsely optimistic picture.

\section{CHOLESTASIS AND PIGMENTATION}

Drug-induced cholestasis is usually an acute disease, but recurrent attacks occur in patients who develop cholestasis as a result of oral contraceptives and pregnancy. There is no evidence to suggest that permanent or progressive liver damage results. However, acute drug cholestasis may occasionally represent the lighting up of an underlying primary biliary cirrhosis. In additon to causing cholestasis, oral contraceptives are associated with an increased incidence of gallstone disease (Boston Collaborative Drug Surveillance Programme, 1973), and there are indeed many different acute and chronic hepatobiliary diseases attributed to contraceptive and anabolic steroids (table). The chronic fibrosing liver disease which occasionally follows unpredictable acute cholestatic drug jaundice (Read et al, 1961; Walker and Combes, 1966) has already been' referred to.

The amount of lipofuscin pigment in liver cells is difficult to quantitate, and the amount varies

\begin{tabular}{ll}
\hline Lesion & Contraceptive or Anabolic \\
\hline Cholestasis & Contraceptive, anabolic \\
Gallstone disease & Contraceptive \\
Peliosis & Anabolic \\
Sinusoidal dilatation & Contraceptive \\
Budd-Chiari syndrome & Contraceptive \\
Liver-cell adenoma & Contraceptive \\
Focal nodular hyperplasia & Contraceptive \\
Liver-cell carcinoma & Anabolic \\
\hline
\end{tabular}

Table Hepatobiliary disorders attributed to contraceptive and anabolic steroids greatly from patient to patient, but Abrahams, Wheatley, Rubenstein, and Stables (1964) demonstrated greatly increased amounts in patients taking phenacetin over long periods, and a similar increase was noted after prolonged chlorpromazine therapy by Greiner and Nicolson (1964).

\section{VASCULAR LESIONS}

Peliosis hepatis, characterized by the formation of cystic blood-filled spaces lined or unlined by endothelial cells, is thought to be caused by treatment with some anabolic steroids (Naeim, Copper, and Semion, 1973). It is not clear whether the initiating factor is vascular dilatation or necrosis. However, a somewhat similar syndrome of periportal vascular dilatation and jaundice has recently been described in patients on oral contraceptives by Poulsen and Winkler (1973), and it may be that the basis of the two lesions is the same. An entirely different way in which contraceptives cause vascular disease is seen when there is thrombosis of hepatic veins leading to the Budd-Chiari syndrome (Sterup and Mosbech, 1967).

Portal hypertension with abnormalities of portal vein branches and in some cases portal fibrosis has been attributed to arsenic therapy given many years before (Viallet, Guillaume, Côté, Légaré, and Lavoie, 1972; Morris, Schmid, Newman, Scheuer, and Sherlock, 1974). One patient described by the latter had large amounts of arsenic in a liver biopsy. The pathogenesis of the lesion is not known, but it is possible that arsenic can damage the walls of the intrahepatic portal vein branches which in these patients were found to be sclerosed or replaced by multiple smaller channels. A similar picture has been reported in patients exposed to vinyl chloride (Marsteller, Lelbach, Müller, Jühe, Lange, Rohner, and Veltman, 1973), and, like vinyl chloride, arsenic has been associated with the development of angiosarcoma (Regelson, Kim, Ospina, and Holland, 1968).

\section{TUMOURS AND TUMOUR-LIKE NODULES}

In addition to the angiosarcomas referred to above, liver-cell tumours have also been attributed to drugs. Baum, Holtz, Bookstein, and Klein (1973) noticed a striking incidence of liver-cell adenoma, usually regarded as rare, in one area of America, and suggested that oral contraceptive agents might have played a role. The patients had been on these for six months to two years. In five of the seven tumours there was rupture or haemorrhage within or from the lesion, and one patient died after a massive intraperitoneal haemorrhage and partial hepatectomy. Contostavlos (1973) described the changes of peliosis hepatis in a similar adenoma, which lends 
support to the idea that the tumours are caused by the steroids. Further case reports have followed, some of them describing the supposedly hamartomatous lesion of focal nodular hyperplasia, with fibrous scars and proliferated bile ducts, rather than pure liver-cell adenoma (Mays, Christopherson, and Barrows, 1974; O’Sullivan and Wilding, 1974).

Lastly, the association has been reported between anabolic steroid therapy in anaemias and tumours histologically resembling liver-cell carcinoma (Bernstein, Hunter, and Yachnin, 1971; Johnson, Feagler, Lerner, Majerus, Siegel, Hartmann, and Thomas, 1972). None of the tumours had metastasized. As in the case of liver-cell adenoma. the lesion of peliosis was present in at least one of the tumours. These tumours, like some of the other lesions described in this paper, are likely to be rare and do not necessarily preclude the use of the incriminated drug. Nevertheless, it seems that chronic and sometimes life-threatening diseases of the liver can be caused by a variety of drugs, and continued vigilance is necessary to detect and prevent their occurrence.

\section{References}

Abrahams, C., Wheatley, A., Rubenstein, A. H., and Stables, D. (1964). Hepatocellular lipofuscin after excessive ingestion of analgesics. Lancet, 2, 621-622.

Baum, J. K., Holtz, F., Bookstein, J. J., and Klein, E. W. (1973). Positive association between benign hepatomas and oral contraceptives. Lancet, 2, 926-929.

Bernstein, M. S., Hunter, R. L., and Yachnin, S. (1971). Hepatomas and peliosis hepatis developing in a patient with Fanconi's anemia. New Engl. J. Med., 284, 1135-1136.

Boston Collaborative Drug Surveillance Programme. (1973). Oral contraceptives and venous thromboembolic disease, surgically confirmed gall bladder disease, and breast tumours. Lancet, 1 , 1399-1404.

Contostavlos, D. L. (1973). Benign hepatomas and oral contraceptives. (Letter) Lancet, 2, 1200.

Cooperative Study (1973). Psoriasis-liver-methotrexate interactions. Arch. Derm., 108, 36-42.

Dahl, M. G. C., Gregory, M. M., and Scheuer, P. J. (1971). Liver damage due to methotrexate in patients with psoriasis. Brit. med. J., 1, 625-630.

Dahl, M. G. C., Gregory, M. M., and Scheuer, P. J. (1972). Methotrexate hepatotoxicity in psoriasis-comparison of different dose regimens. Brit. med. J., 1, 654-656.

Davidson, D. G. D., and Eastham, W. N. (1966). Acute liver necrosis following overdose of paracetamol. Brit. med. J., 2, 497-499.

Fog, C. V. M. (1949). On protein intake, hepatitis and consumption of laxatives. Acta. med. scand., Suppl., 234, 125-129.

Franklin, M., Bean, W. B., and Hardin, R. C. (1950). Fowler's solution as an etiologic agent in cirrhosis. Amer. J. med. Sci., 219, 589-596.
Goldstein, G. B., Lam, K. C., and Mistilis, S. P. (1973). Drug-induced active chronic hepatitis. Amer. J. dig. Dis., 18, 177-184.

Greiner, A. C., and Nicolson, G. A. (1964). Pigment deposition in viscera associated with prolonged chlorpromazine therapy. Canad. med. Ass. J., 91, 627-635.

Ishak, K. G., and Irey, N. S. (1972). Hepatic injury associated with the phenothiazines. Arch. Path., 93, 283-304.

Johnson, F. L., Feagler, J. R., Lerner, K. G., Majerus, P. W., Siegel, M., Hartmann, J. R., and Thomas, E. D. (1972). Association of androgenic-anabolic steroid therapy with development of hepatocellular carcinoma. Lancet, 2, 1273-1276.

Klatskin, G. (1969). Toxic and drug-induced hepatitis. In Diseases of the Liver, 3rd ed., edited by L. Schiff, pp. 498-601. Lippincott, Philadelphia.

Klatskin, G., and Kimberg, D. V. (1969). Recurrent hepatitis attributable to halothane sensitization in an anesthetist. New Engl. J. Med., 280, 515-522.

McHardy, G., and Balart, L. A. (1970). Jaundice and oxyphenisatin. J. Amer. med. Ass., 211, 83-85.

Marsteller, H. J., Lelbach, U. K., Müller, R., Jühe, S., Lange, C. E., Rohner, H. G., and Veltman, G. (1973). Chronisch-toxische Leberschäden bei Arbeitern in der PVC Produktion Dtsch. med. Wschr., 98, 2311-2314.

Mays, E. T., Christopherson, W. M., and Barrows, G. H. (1974). Focal nodular hyperplasia of the liver. Possible relationship to oral contraceptives. Amer. J. clin. Path., 61, 735-746.

Merritt, A. D., and Fetter, B. F. (1959). Toxic hepatic necrosis (hepatitis) due to isoniazid: Report of a case with cirrhosis and death due to haemorrhage from esophageal varices. Ann. intern. Med., 50, 804-810.

Morris, J. S., Schmid, M., Newman, S., Scheuer, P. J., and Sherlock, S. (1974). Arsenic and non-cirrhotic portal hypertension. Gastroenterology, 66, 86-94.

Naeim, F., Copper, P. H., and Semion, A. A. (1973). Peliosis hepatis. Possible etiologic role of anabolic steroids. Arch. Path., 95, 284-285.

O'Sullivan, J. P., and Wilding, R. P. (1974). Liver hamartomas in patients on oral contraceptives. Brit. med. J., 3, 7-10.

Poulsen, H., and Winkler, K. (1973). Liver disease with peripheral sinusoidal dilatation. (Abst.) Digestion, 8, 441-442.

Read, A. E., Harrison, C. V., and Shelock, S. (1961). Chronic chorpromazine jaundice with particular reference to its relationship to primary biliary cirrhosis. Amer. J. Med., 31, 249-258.

Regelson, W., Kim, U., Ospina, J., and Holland, J. F. (1968). Hemangioendothelial sarcoma of liver from chronic arsenic intoxication by Fowler's solution. Cancer (Philad.), 21, 514-522.

Reynolds, T. B., Lapin, A. C., Peters, R. L., and Yamahiro, H. S. (1970). Puzzling jaundice. Probable relationship to laxative ingestion. J. Amer. med. Ass., 211, 86-90.

Reynolds, T. B., Peters, R. L., and Yamada, S. (1971). Chronic active and lupoid hepatitis caused by a laxative, oxyphenisatin. New Engl. J. Med., 285, 813-820.

Schweitzer, I. L., and Peters, R. L. (1974). Acute submassive hepatic necrosis due to methyldopa. Gastroenterology, 66, 1203-1211.

Seaman, W. E., Ishak, K. G., and Plotz, P. H. (1974). Aspirin-induced hepatotoxicity in patients with systemic lupus erythematosus. Ann. intern. Med., 80, 1-8.

Sterup, K., and Mosbech, J. (1967). Budd-Chiari syndrome after taking oral contraceptives. Brit. med. J., 4, 660.

Tönder, M., Nordöy, A., and Elgjo, K. (1974). Sulfonamide-induced chronic liver disease. Scand. J. Gastroent., 9, 93-96.

Viallet, A., Guillaume, E., Côté, J., Légaré, A., and Lavoie, P. (1972) Presinusoidal portal hypertension following chronic arsenic intoxication. (Abst.) Gastroenterology, 62, 177.

Walker, C. O., and Combes, B. (1966). Biliary cirrhosis induced by chlorpromazine. Gastroenterology, 51, 631-640. 